\title{
Local Antimagic Vertex Coloring of Gear Graph
}

\author{
Masdaria Natalina Br Silitonga, Kiki Ariyanti Sugeng*
}

\author{
Department of Mathematics, Faculty of Mathematics and Sciences \\ Universitas Indonesia, Depok 16424, Indonesia \\ *Corresponding author.Email: kiki@sci.ui.ac.id
}

\begin{abstract}
Let $G=(V, E)$ be a graph that consist of a vertex set $V$ and an edge set $E$. The local antimagic labeling $f$ of a graph $G$ with edge-set $E$ is a bijection map from $E$ to $\{1,2, \ldots,|E|\}$ such that $w(u) \neq w(v)$, where $w(u)=\sum_{e \in E(u)} f(e)$ and $E(u)$ is the set of edges incident to $u$. In this labeling, every vertex $v$ is assigned $w(v)$ as its color. The minimum number of colors in a local antimagic labelling, is called a local antimagic chromatic number and denoted by $\chi_{l a}(G)$. This paper contribution is to determine the local antimagic chromatic number $\chi_{l a}\left(G_{n}\right)$ of a gear graph. A gear graph is a graph obtained by inserting additional vertex between each pair of adjacent vertices on the circumference of the wheel graph $W_{n}$. The gear graph $G_{n}$ has $2 n+1$ vertices and $3 n$ edges.
\end{abstract}

Keywords: Antimagic labeling, Local antimagic labeling, Local antimagic chromatic number, Gear graph.

\section{INTRODUCTION}

Graphs are used in various fields, such as network topology modeling, database design, scheduling, traveling salesman problems, and so on [1]. Graph labeling is one of the interesting research topics in graph theory that assign an element of graph such as vertices and edges with the set of integers called labels [2].

Formally, the labeling of antimagic in a graph is defined as follows. Suppose $G=(V, E)$ be a simple undirected graph with a non-empty set of vertices $V$ and a set of edges $E$. The number of vertices, denoted by $|V(G)|$, referred to the order of $G$ and the number of edges, denoted by $|E(G)|$, called size of $G$ [3]. If vertex $v$ is an endpoint of edges $e$, then $v$ is said to be incident on $e$ and $e$ is incident on $v$. Two vertices are adjacent if they are the endvertices of the same edge [4]. The local antimagic labeling $f$ of a graph $G$ with edge-set $E$ is a bijection map from $E$ to $\{1,2, \ldots,|E|\}$ such that $w(u) \neq$ $w(v)$, where $w(u)=\sum_{e \in E(u)} f(e)$ and $E(u)$ is the set of edges incident to $u$, for $u, v \in V(G)$. A graph $G$ is called local antimagic if $G$ has a local antimagic labeling [5]. The local antimagic chromatic number of $G$, denoted by $\chi_{l a}(G)$, is the minimum number of colors that is needed that is induced by local antimagic labelings of $G$ [6].

Arumugam, et al., [7] presented local antimagic chromatic number of several classes of graphs such as complete graph $K_{n}$ for $n \geq 3, \chi_{\text {la }}\left(K_{n}\right)=n$; star graph $K_{1, n}$ for $n \geq 3, \chi_{l a}\left(K_{1, n-1}\right)=n$; path graph $P_{n}$ for $n \geq$ $3, \chi_{l a}\left(P_{n}\right)=3$; cycle graph $C_{n}$ for $n \geq 3, \chi_{l a}\left(C_{n}\right)=3$; friendship graph $F_{n}$ for $n \geq 2, \chi_{l a}\left(F_{n}\right)=3$; complete bipartite graph $K_{m, n}$ for $m, n \geq 2, \chi_{l a}\left(K_{m, n}\right)=2$ if only if $m \equiv n(\bmod 2)$ and wheel graph $W_{n}$ with $n+1$ order for $n \geq 3, \chi_{l a}\left(W_{n}\right)=4$, if $n \equiv 1,3(\bmod 4)$, $\chi_{l a}\left(W_{n}\right)=3$, if $n \equiv 2(\bmod 4)$, and $3 \leq \chi_{l a}\left(W_{n}\right) \leq$ $n$ if $n \equiv 0(\bmod 4)$. Furthermore, Pratama, et al., [8] presented the local super antimagic total chromatic number of several classes of graph which is related with wheel graph, such as fan graph $F_{n}$ for $n \geq 3,3 \leq$ $\chi_{\text {lsat }}\left(F_{n}\right) \leq 4$; even gear graph $G_{n}$ for $n \geq 4,2 \leq$ $\chi_{\text {lsat }}\left(G_{n}\right) \leq 3$; odd sun flower graph $S F_{n}$ for $n \geq 5$, $4 \leq \chi_{\text {lsat }}\left(S F_{n}\right) \leq 5$.

We study and determine the local antimagic chromatic numbers of a gear graph in this paper.

\section{MAIN RESULT}

We begin this section by presenting the gear graph as follow. Gear graph, denoted by $G_{n}$ (Figure 1) is a graph obtained from wheel graph $W_{n}$ by adding a vertex between each pair of adjacent rim vertices [9]. Clearly $G_{n}$ has a set of vertices $V=\left\{c, v_{i}, u_{i} ; 1 \leq i \leq n\right\}$ and 
set of edges $E=\left\{c v_{i}, v_{i} u_{i} ; 1 \leq i \leq n\right\} \cup$ $\left\{u_{i} v_{i+1} ; 1 \leq i \leq n-1\right\} \cup\left\{u_{n} v_{1}\right\}$ such that $|V|=$ $2 n+1$ and $|E|=3 n$.

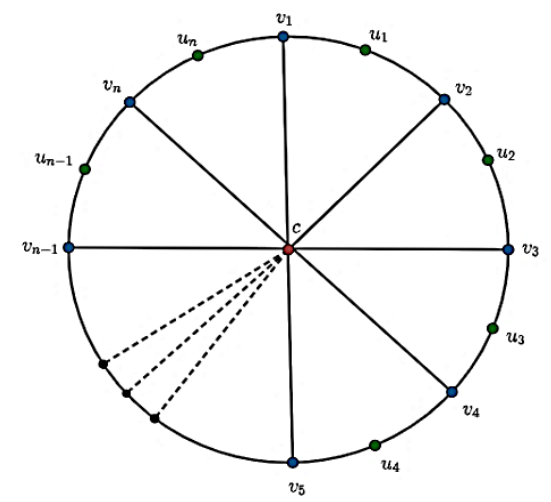

Figure 1 Gear Graph $G_{n}$.

\subsection{Local Antimagic Chromatic Number of $G_{n}$ for $n$ odd}

Lemma 2.1.1. gives the upper bound of $\chi_{l a}\left(G_{n}\right)$ for $n \equiv 1(\bmod 2)$.

Lemma 2.1.1. $X_{l a}\left(G_{n}\right) \leq 4$ for $n \equiv 1(\bmod 2)$ and $n \geq 3$.

\section{Proof:}

Case 1: for $n=3$.

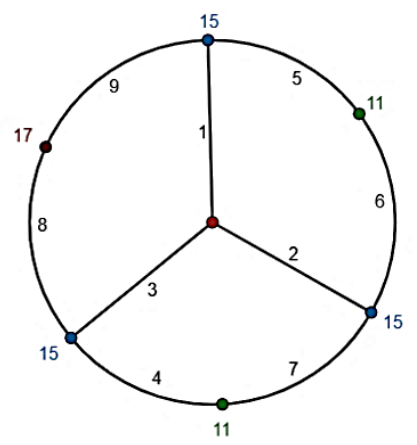

Figure $2 \chi_{1 a}\left(G_{3}\right)=4$.

Case 2: for $n \equiv 1(\bmod 2)$ and $n \geq 5$.

Label the edges $c v_{i}$, for $i=1,2, \ldots, n$, as follows:

$$
f\left(c v_{i}\right)= \begin{cases}1, & \text { for } i=1, \\ n-i+2, & \text { for } i \text { odd, } i \neq 1, \\ i+1, & \text { for } i \text { even. }\end{cases}
$$

Label the edges $v_{i} u_{i}$, for $i=1,2, \ldots, n$, as follows:

$$
f\left(v_{i} u_{i}\right)= \begin{cases}\frac{3 n+i}{2}, & i \text { odd } \\ \frac{6 n-i}{2}, & i \text { even. }\end{cases}
$$

Label the edges $u_{i} v_{i+1}$, for $i=1,2, \ldots, n-1$, as follows:

$$
f\left(u_{i} v_{i+1}\right)= \begin{cases}\frac{3 n-i}{2}, & i \text { odd, } \\ \frac{4 n+i}{2}, & i \text { even. }\end{cases}
$$

Label the edges $u_{n} v_{1}$, as follows:

$$
f\left(u_{n} v_{1}\right)=3 n \text {. }
$$

In Figure 3, we have the labeling of the edges $G_{n}$ for $n$ odd.

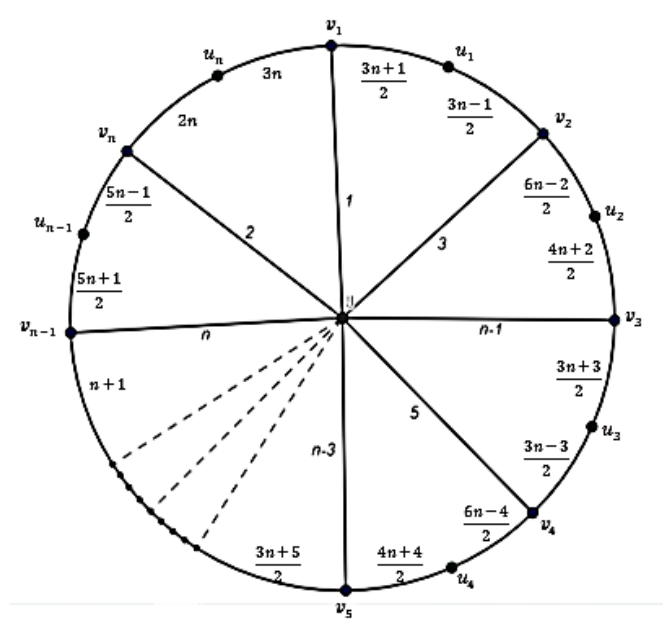

Figure 3 Labeling Edges $G_{n}$ for $n$ odd.

This labelling provides different weights for any two adjacent vertices, namely:

$$
\begin{gathered}
w(c)=\frac{1}{2} n(n+1), \\
w\left(v_{i}\right)=\frac{1}{2}(9 n+3),
\end{gathered}
$$

$$
w\left(u_{i}\right)=\left\{\begin{array}{cc}
3 n, & \text { for } 1 \leq i<n, i \text { odd, } \\
5 n, & \text { for } 1<i<n, i \text { even, } \\
i=n .
\end{array}\right.
$$

Thus, the labeling gives 4 different weights, that is, $x_{l a}\left(G_{n}\right) \leq 4$, for $n \equiv 1(\bmod 2)$ and $n \geq 3$. 
In Figure 4, we have example of labeling $G_{7}$. We now prove the lower bound of the local antimagic chromatic number of $G_{n}$ for $n$ is odd.

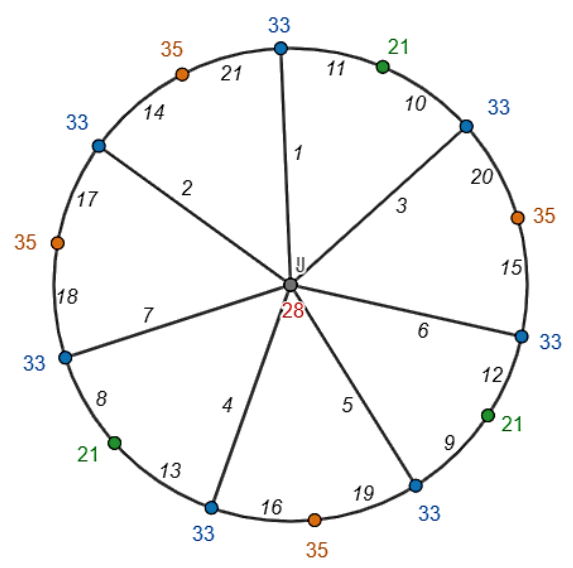

Figure 4 Labeling of Graph $G_{7}$.

Lemma 2.1.2. $\chi_{l a}\left(G_{n}\right) \geq 3$ for $n \equiv 1(\bmod 2)$ and $n \geq 3$.

Proof: Suppose that $\chi_{l a}\left(G_{n}\right)=2$ with $w\left(v_{i}\right) \neq$ $w\left(u_{i}\right)$. Then there is a labeling $c v_{i}$ for $1 \leq i \leq n$ such that $w(c)=w\left(u_{i}\right)$. The largest $w\left(v_{i}\right)$ cannot be more than the sum of the edge labels labeled $n$ and two largest edge labels, that is, $w\left(v_{i}\right)=n+\frac{5 n+1}{2}+(n+1)=$ $\frac{9 n+3}{2}$. While the weight of the central vertex must be at least the sum of the smallest $n$ edge labels, that is, $w(c)=1+2+\cdots+n=\frac{1}{2} n(n+1)$. It is clear that no $n \in \mathbb{N}$ satisfies the equation $9 n+3=n(n+1)$, this is a contradiction. So, it must be $\chi_{l a}\left(G_{n}\right) \geq 3$ for $n \equiv$ $1(\bmod 2)$.

Theorem 2.1. The local antimagic chromatic number of $G_{n}$ for $n$ odd and $n \geq 3$ satisfies $3 \leq \chi_{l a}\left(G_{n}\right) \leq 4$.

Proof: Based on Lemma 2.1.1. and Lemma 2.1.2., it is proven that $3 \leq \chi_{l a}\left(G_{n}\right) \leq 4$.

\subsection{Local Antimagic Chromatic Number of $G_{n}$ for $n$ even}

Lemma 2.2.1. gives the upper bound of $\chi_{l a}\left(G_{n}\right)$ for $n \equiv 0(\bmod 2)$.
Lemma 2.2.1. $x_{l a}\left(G_{n}\right) \leq 4$ for $n \equiv 0(\bmod 2)$ and $n \geq 4$.

Proof: For $n \equiv 0(\bmod 2)$ and $n \geq 4$.

Label the edges $c v_{i}$, for $i=1,2, \ldots, n$, as follows:

$$
f\left(c v_{i}\right)=\left\{\begin{array}{lr}
i, & 1 \leq i \leq \frac{n}{2}, i \text { odd, } \\
n-i+1, & 1 \leq i \leq \frac{n}{2}, i \text { even, } \\
n, & i=\frac{n}{2}+1, \\
i-1, & \frac{n}{2}+1<i \leq n, i \text { odd }, \\
n-i+2, & \frac{n}{2}+1<i \leq n, i \text { even. }
\end{array}\right.
$$

Label the edges $v_{i} u_{i}$, for $i=1,2, \ldots, n$, as follows:

$$
f\left(v_{i} u_{i}\right)=\left\{\begin{array}{cc}
\frac{4 n-i+1}{2} & 1 \leq i \leq \frac{n}{2}, i \text { odd } \\
\frac{3 n+i}{2} & 1 \leq i \leq \frac{n}{2}, i \text { even } \\
\frac{3 n-i+1}{2} & \frac{n}{2}<i \leq n, i \text { odd } \\
\frac{2 n+i}{2} & \frac{n}{2}<i \leq n, i \text { even. }
\end{array}\right.
$$

Label the edges $u_{i} v_{i+1}$, for $i=1,2, \ldots, n-1$, as follows:

$$
f\left(u_{i} v_{i+1}\right)= \begin{cases}\frac{4 n+i+1}{2} & 1 \leq i \leq \frac{n}{2}, i \text { odd. } \\ \frac{5 n-i+2}{2} & 1 \leq i \leq \frac{n}{2}, i \text { even } \\ \frac{5 n+i+1}{2} & \frac{n}{2}<i \leq n, i \text { odd } \\ \frac{6 n-i+2}{2} & \frac{n}{2}<i \leq n, i \text { even. }\end{cases}
$$

Label the edges $u_{n} v_{1}$, as follows:

$$
f\left(u_{n} v_{1}\right)=\frac{5 n+2}{2}
$$

In Figure 5, we have labeling the edges $G_{n}$ for $n$ even.

This labelling formula gives us four different values of vertex weight as follows.

$$
w(c)=\frac{1}{2} n(n+1),
$$




$$
w\left(v_{i}\right)=\left\{\begin{array}{cl}
\frac{9 n+4}{2}, & 1 \leq i \leq \frac{n}{2}, i \text { odd } \\
& \frac{n}{2} \leq i \leq n, i \text { even, } \\
\frac{9 n+2}{2}, & 1 \leq i \leq \frac{n}{2}, i \text { odd } \\
& \frac{n}{2} \leq i \leq n, i \text { even, }
\end{array}\right.
$$

Thus, $X_{l a}\left(G_{n}\right) \leq 4$, for $n \equiv 0(\bmod 2)$ and $n \geq 4$.

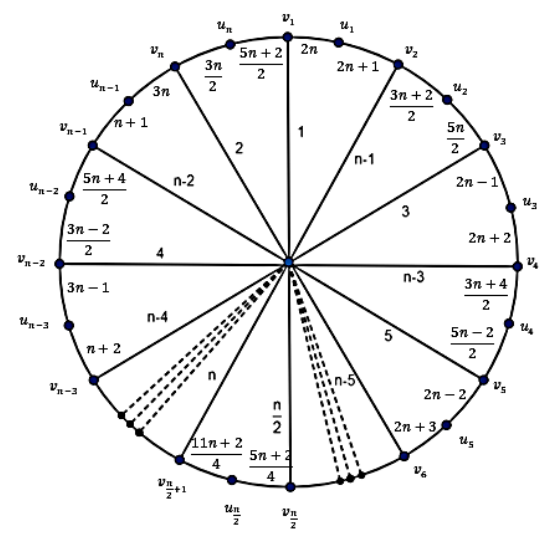

Figure 5 Labeling Edges $G_{n}$ for $n$ even.

In Figure 6, we have example of labeling $G_{8}$.

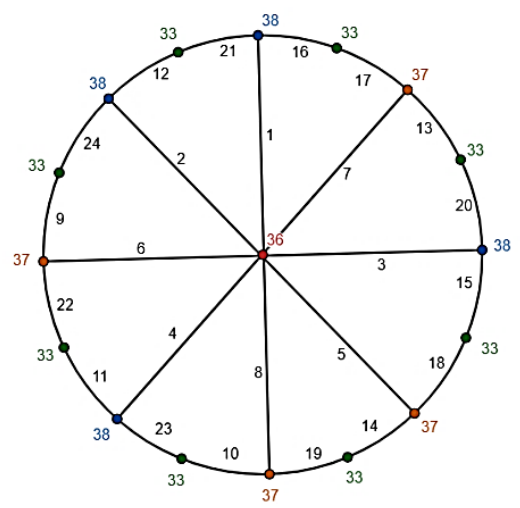

Figure 6. Labeling of $G_{8}$.

In Lemma 2.2.2, we show the lower bound of the local antimagic chromatic number of $G_{n}$ for $n$ is even.

Lemma 2.2.2. $X_{l a}\left(G_{n}\right) \geq 4$ for $n \equiv 0(\bmod 2)$ and $n \geq 4$.

Proof: From Lemma 2.1.2. it has been proved that $\chi_{l a}\left(G_{n}\right) \geq 3$ for $n$ is odd. Suppose for $n$ is even $\chi_{l a}\left(G_{n}\right)=3$. Similar to the proof of Lemma 2.1.2, the weights are $w(c) \neq w\left(v_{i}\right)$. Since vertices $u_{i}$ and $v_{i}$ are neighbors, they must be $w\left(u_{i}\right) \neq w\left(v_{i}\right)$. Suppose the weight of each vertex $v_{i}$ is the same. Because every vertex $v_{i}$ is adjacent to edge $c v_{i}, v_{i} u_{i}$, and $u_{i-1} v_{i}$ (or $u_{n} v_{1}$, if $i=1$ ), a labeling of all edges $G_{n}$ will be constructed so that each vertex $v_{i}$ has the same weight. It is known that the sum of all edge labels $G_{n}$ is $\sum_{i=1}^{3 n} i=$ $\frac{3 n}{2}(3 n+1)$, in this case there is no integer for even $n$ that can evenly divide the sum of all edge labels $G_{n}$. This results in at least two vertices $v_{i}$ has different weights. This is a contradiction, so it must be $\chi_{l a}\left(G_{n}\right) \geq$ 4 for $n \equiv 0(\bmod 2)$.

Theorem 2.2. The local antimagic chromatic number of $G_{n}$ for $n$ even and $n \geq 4$ is $X_{l a}\left(G_{n}\right)=4$.

Proof: Based on Lemma 2.2.1. and Lemma 2.2.2., it is proven that $x_{l a}\left(G_{n}\right)=4$.

\section{CONCLUSION}

We proved the the local antimagic chromatic number of gear graph $\chi_{l a}\left(G_{n}\right)$. We propose an open problem: What is the local antimagic chromatic number for other graph that is also related to wheel graph?

\section{AUTHORS' CONTRIBUTIONS}

Conceptualization, M.N.S., K.A.S.; proof methodology, M.N.S., K.A.S.; writing-original draft preparation, M.N.S, K.A.S.; writing-review and editing, M.N.S., K.A.S.; funding acquisition, K.A.S. All authors have read and agreed to the published version of the manuscript.

\section{ACKNOWLEDGMENTS}

This research has supported by PUTI KIUniversitas Indonesia 2020 Research Grant No. NKB779/UN2.RST/HKP.05.00/2020.

\section{REFERENCES}

[1] A. Elumalai, Graph coloring and labelling applications in computer sciences, in: Malaya Journal of Matematic, Vol. S, No.2, 2039-4041, 2020.

DOI: https://doi.org/10.26637/MJM0S20/1048

[2] S. N. Daoud, Edge odd graceful labelling of some path and cycle related graphs, in: AKCE International Journal of Graphs and Combinatoric, 
vol. 4, 2017, pp. 178-203. DOI: http://dx.doi.org/10.1016/j.akcej.2017.03.001

[3] P. J. Slater, It is all labeling, in: R. Gera et al. (eds), Graph Theory, Problem Books in Mathematics, Springer, 2016. DOI: 10.1007/978-3-319-319407_14

[4] J. L. Gross and Jay Yellen, Fundamentals of Graph Theory, In: Gross et al. (eds). Handbook of Graph Theory second edition, CRC Press, 2014. DOI: 978-1-4398-8019-7

[5] S. Arumugam, Yi-Chun Lee, K. Premalatha, TaoMing Wang, On Local Antimagic Vertex Coloring for Corona Products of Graphs, in Journal Mathematics Combinatoric, 2018. ArXiv: 1808.04956v1.

[6] S. Slamin, N.O Adiwijaya, M.A. Hasan, D. Dafik, K. Wijaya, Local super antimagic total labeling for vertex coloring of graphs, In Journal Symmetry, vol. 12, 2020. DOI: 10.3390/sym12111843

[7] S. Arumugam, K. Premalatha, M. Baca, and A. Semaničová-Feňovčíková, Local antimagic vertex coloring of a graphs, Graph and Combinatorics, Vol. 33, PP. 275-285. DOI: 10.1007/s00373-0171758-7

[8] S.A. Pratama, S. Setiawan, S. Slamin, Local super antimagic total vertex coloring of some wheel related graphs, Journal of Physics: conference series 2020, 1538, 012014. DOI: 10.1088/1742$6596 / 1538 / 1 / 012014$

[9] V.R. Kulli, Some new Kulli-Basava topological indices, in: Earthline Journal of Mathematical Sciences, vol. 2, no. 2, October 2019, pp. 343-354. DOI: https://doi.org/10.34198/ejms.2219.343354 